\title{
Distortion Theorems for the Level Curves of Rational Functions and Harmonic Functions
}

\section{DOROTHY BROWNE SHAFFER}

\author{
Communicated by J. B. Diaz
}

I. Introduction. The level loci of Green's function and more general harmonic functions have certain properties of smoothness and limitations on distortion. These properties of the level curves and orthogonal trajectories of Green's function, including multiple points, tangents, normals and convexity have been studied by J. L. Walsh in a series of publications [4, 5, 6]. In a recent paper [3] the author studied the distortion and location of the circle of curvature of lemniscates and the level curves of Green's function. It is the object of this paper to extend these results to general rational functions and harmonic functions. The level loci of a harmonic function, are by no means arbitrary as to their shape, but also have certain limitations on distortion. The present investigation establishes best possible geometric bounds for these properties for rational functions, all of whose zeros and poles are contained in disjoint discs. The same bounds are shown to be valid for functions harmonic in a region bounded by sets of curves $C_{1 i}, i=1, \cdots, m$ and $C_{2 i}, i=1, \cdots, n ; u(x, y)=1$ on the set $C_{1 i} \subset \operatorname{disc} D_{1}$ and $u(x, y)=0$ on $C_{2 i} \subset \operatorname{disc} D_{2}$.

II. Normals and bisectors. The foundation for the theory of the critical points of a rational function of a complex variable $z$ :

$$
R(z)=\frac{\prod_{i=1}^{m}\left(z-\alpha_{i}\right)}{\prod_{i=1}^{n}\left(z-\beta_{i}\right)}
$$

was provided by Bôcher [1] who modified the field of force in Gauss' theorem by admitting both positive and negative masses. The lines of force are the loci $\arg R(z)=k$ which are orthogonal to the level curves $R_{\mu}$ :

$$
|R(z)|=\frac{\prod_{i=1}^{m}\left|z-\alpha_{i}\right|}{\prod_{i=1}^{n}\left|z-\beta_{i}\right|}=\mu>0 .
$$


Our first result is a generalization of Theorem 1 in [3]. (The result was obtained by a different method, for lemniscates only, by Davis and Pollack [2]).

Theorem 1. The perpendicular bisector of a chord of a level curve (2) cannot separate the finite zeros and finite poles of (1).

Proof. Suppose the contrary. Let $z_{a}$ and $z_{b}$ lie on (2). Assume all the zeros $\alpha_{i}, i=1, \cdots, m$, to lie to the same side of the bisector as $z_{a}$, and all the poles $\beta_{i}, i=1, \cdots, n$, to the opposite side, then

$$
\frac{\prod_{i=1}^{m}\left|z_{a}-\alpha_{i}\right|}{\prod_{i=1}^{n}\left|z_{a}-\beta_{i}\right|}<\frac{\prod_{i=1}^{m}\left|z_{b}-\alpha_{i}\right|}{\prod_{i=1}^{n}\left|z_{b}-\beta_{i}\right|}
$$

and $z_{a}$ and $z_{b}$ cannot lie on (2).

If we let $z_{a}$ and $z_{b}$ approach each other and coincide at $P$, we obtain:

Theorem 2. The normal to the level curve (2) at $P$ cannot separate the finite zeros and poles of (1).

Further results can be deduced by specializing to the case that all zeros and poles of a rational function are contained in disjoint discs. In the following corollary the possibility that all the poles and zeros lie to one side of the bisector is ruled out.

Corollary 1. Let all $\alpha_{i} \subset D_{1}, i=1, \cdots, n$ and all $\beta_{i} \subset D_{2}, i=1, \cdots, n$, $D_{1} \cap D_{2}=\varnothing$; then the normal to (2) at the internal center of similitude of the two circles $C_{1}$ and $C_{2}$ bounding $D_{1}$ and $D_{2}$ must intersect $D_{1}$ and $D_{2}$.

The configuration of Corollary 1 is invariant under linear transformations and we obtain the following result due to Walsh [4, p. 277].

Corollary 2. Let $\alpha_{i} \subset D_{1}, i=1, \cdots, n$ and $\beta_{i} \subset D_{2}, i=1, \cdots, n, D_{1} \cap D_{2}=$ $\varnothing$; then the normal to (2) at a point $P$ exterior to $D_{1} \cup D_{2}$ must lie in the circular sector bounded by the two circles through $P$ which are transversely tangent to $C_{1}$ and $C_{2}$.

III. Curvature. Without loss of generality we can choose the coordinate system so that the point $P(x, y)$ is the origin, and let the normal to the level curve (2) coincide with the negative $y$-axis. By writing equation (1) in rectangular coordinates and taking the logarithmic derivative, and substituting in the usual formula for the curvature $K$, we obtain for the curvature $K$ of $R_{\mu}$ at $P(x, y)=(0,0)$ :

$$
K=-\frac{R_{x x}}{R_{y}}=\frac{\sum_{i=1}^{m}\left(\frac{1}{r_{i}^{2}}-\frac{2 x_{i}^{2}}{r_{i}^{4}}\right)-\sum_{i=1}^{n}\left(\frac{1}{R_{i}^{2}}-\frac{2 X_{i}^{2}}{R_{i}^{4}}\right)}{\sum_{i=1}^{m} \frac{y_{i}}{r_{i}^{2}}-\sum_{i=1}^{n} \frac{Y_{i}}{R_{i}^{2}}}
$$




$$
\begin{array}{lll}
\alpha_{i}=x_{i}+i y_{i}, & \left|\alpha_{i}\right|=r_{i}, & i=1, \cdots, m, \\
\beta_{i}=X_{i}+i Y_{i}, & \left|\beta_{i}\right|=R_{i}, & i=1, \cdots, n .
\end{array}
$$

This choice of coordinate system and notation will be used throughout. Our first result on the curvature of $R_{\mu}$ is the following:

Theorem 3. Let all the zeros $\alpha_{i}$ lie in a half plane $Q_{1}$, boundary $q_{1}$ and the poles $\beta_{i}$ lie in a half plane $Q_{2}$, boundary $q_{2}, q_{2}$ parallel to $q_{1}, Q_{1} \cap Q_{2}=\varnothing$. Let $P$ be an arbitrary point in the strip between $Q_{1}$ and $Q_{2}$. Denote by $n$ the normal to (2) at $P$. Let $A_{1}=n \cap q_{1}$, and $A_{2}=n \cap q_{2}$. Then the radius of curvature of (2) at $P$ is numerically greater than the smaller of the distances $P A_{1}$ and $P A_{2}$.

Proof. Write the equations for $Q_{1}$ and $Q_{2}$ in the form $y>a x+b, b>0$, and $Y<a X-B, B>0$. It follows that:

$$
-R_{y}>b \sum_{i=1}^{m} \frac{1}{r_{i}^{2}}+B \sum_{i=1}^{n} \frac{1}{R_{i}^{2}} .
$$

Without loss of generality assume $b<B$; then we wish to prove $|K|<1 / b$.

The proof is divided into two cases, $K>0$, i.e. $R_{x x}>0$, and $K<0$, i.e. $R_{x x}<0$, and the desired result follows in each case from substitution in (3).

Our main result concerns the precise upper and lower bounds for $K$ when the $\alpha_{i}$ lie in a disc $D_{1}$ and the $\beta_{i}$ lie in a disc $D_{2}, D_{1} \cap D_{2}=\varnothing$. This theorem is an extension of the central result of [2, Th. 3, pp. 61-65] which states the bounds for the curvature of lemniscates whose poles are contained in a disc.

Theorem 4. Let $R_{\mu}$ be the level curve of a rational function (2) whose zeros $\alpha_{i}$ lie in a disc $D_{1}$ and whose poles $\beta_{i}$ lie in a disc $D_{2}, D_{1} \cap D_{2}=\varnothing$. Let $P$ be an arbitrary point exterior to $D_{1} \cup D_{2}$. Draw the four circles through $P$ tangent to $D_{1}$ or $D_{2}$, and orthogonal to the normal $n$ to (2) at $P$. Let $A$ and $B$ denote the intersections of the circles tangent to $D_{1}$ with $n$, and $C$ and $D$ the intersections of the circles tangent to $D_{2}$ with $n$. Let the directed distances $P A, P B, P C$, and $P D$ be denoted by $1 / a, 1 / b, 1 / c$, and $1 / d, a \leqq b, d \leqq c$. Then the following bounds are obtained for the curvature $K$ of (2):

(4a) $K \leqq K_{\max }=b+c$,

$$
\text { for } b-d \leqq 2(b-c) \text {, }
$$

(4b) $K \leqq K_{\max }=4 b-d-c-2[2(b-c)(b-d)]^{1 / 2}$, for $b-d \geqq 2(b-c)$,

(4c) $K \geqq K_{\min }=d+a$, for $b-d \leqq 2(a-d)$,

(4d) $K \geqq K_{\min }=4 d-a-b+2[2(a-d)(b-d)]^{1 / 2}$, for $b-d \geqq 2(a-d)$.

The proof is based on a study of a rational function with only one pair of zeros and one pair of poles; the extremal configuration is then reduced to that case.

In the following lemma the bounds (4a) to (4d) are established for this special case. 
Lemma 1. For the set of level curves

$$
\begin{gathered}
|R(z)|=\frac{\left(z-\alpha_{1}\right)^{m_{1}}\left(z-\alpha_{2}\right)^{m_{2}}}{\left(z-\beta_{1}\right)^{n_{1}}\left(z-\beta_{2}\right)^{n_{2}}}, \\
m_{1}+m_{2}=n_{1}+n_{2}=n, \quad m_{1}>0, \quad m_{2}>0, \quad n_{1}>0, \quad n_{2}>0 ; \\
\alpha_{1} \subset D_{1}, \quad \alpha_{2} \subset D_{1} ; \quad \beta_{1} \subset D_{2}, \quad \beta_{2} \subset D_{2} ; \quad D_{1} \cap D_{2}=\varnothing ;
\end{gathered}
$$

the bounds (4a), (4b), (4c), and (4d) hold.

Proof. In the proof of Iemma 1 we will invert the $z$-plane in the unit circle. This device allows simplified formulae for the first and second derivatives of (2) in terms of new coordinates; and a greatly simplified geometric situation in the inverted plane allows easier determination of the extremal configurations.

If we now denote by $\left(x_{1}, y_{1}\right)$ and $\left(x_{2}, y_{2}\right)$ the coordinates of the zeros after inversion and by $\left(X_{1}, Y_{1}\right)$ and $\left(X_{2}, Y_{2}\right)$ those of the poles after inversion we obtain for the curvature of (5):

$$
\begin{gathered}
K=\frac{m_{1}\left(y_{1}^{2}-x_{1}^{2}\right)+m_{2}\left(y_{2}^{2}-x_{2}^{2}\right)-n_{1}\left(Y_{1}^{2}-X_{1}^{2}\right)-n_{2}\left(X_{2}^{2}-Y_{2}^{2}\right)}{m_{1} y_{1}+m_{2} y_{2}-n_{1} Y_{1}-n_{2} Y_{2}}, \\
\alpha_{1} \text { and } \alpha_{2} \text { lie on the boundary } C_{1} \text { of } D_{1} \text { and } \\
\beta_{1} \text { and } \beta_{2} \text { lie on the boundary } C_{2} \text { of } D_{2} .
\end{gathered}
$$

Let

$$
\begin{aligned}
& m_{1} x_{1}+m_{2} x_{2}=n_{1} X_{1}+n_{2} X_{2}=n x_{0}, \\
& n s_{1}=m_{1} y_{1}+m_{2} y_{2}, \quad s_{1}=M G_{1}, \quad s_{2}=M G_{2}, \\
& n s_{2}=n_{1} Y_{1}+n_{2} Y_{2} .
\end{aligned}
$$

$M$ is the point $(x, 0), G_{1}$ is the center of gravity of the two zeros $Z_{1}$ and $Z_{2}$; and $G_{2}$ that of the poles $P_{1}$ and $P_{2} . \theta_{1}$ is the angle between the $x$-axis and the line joining $Z_{1} Z_{2} ; \theta_{2}$ is the angle between the $x$-axis and the line joining $P_{1} P_{2}$. Substituting in (6)

$$
K=\frac{s_{1}^{2}-\overline{Z_{1} G_{1}} \cdot \overline{Z_{2} G_{1}} \cos 2 \theta_{1}-\left(s_{2}^{2}-\overline{P_{1} G_{2}} \cdot \overline{P_{1} G_{2}} \cos 2 \theta_{2}\right)}{s_{1}-s_{2}} .
$$

For our choice of coordinate system $\left(s_{1}-s_{2}\right)>0$. Then if we keep $s_{1}$ and $s_{2}$ fixed and vary $\theta_{1}$ and $\theta_{2}$

$$
\begin{aligned}
& K \leqq \frac{s_{1}^{2}+\overline{Z_{1} G_{1}} \cdot \overline{Z_{2} G_{1}}-s_{2}^{2}+\overline{P_{1} G_{2}} \cdot \overline{P_{2} G_{2}}}{s_{1}-s_{2}}=f_{1}\left(s_{1}, s_{2}\right), \\
& K \geqq \frac{s_{1}^{2}-\overline{Z_{1} G_{1}} \cdot \overline{Z_{2} G_{1}}-s_{2}^{2}-\overline{P_{1} G_{2}} \cdot \overline{P_{2} G_{2}}}{s_{1}-s_{2}}=f_{2}\left(s_{1}, s_{2}\right) .
\end{aligned}
$$

It follows from (9) and (10) that the curvature of a level curve (5) with the poles and zeros in the interior of the discs $D_{1}$ and $D_{2}$ will lie within the bounds 
established for the level curve (5) with the poles and zeros on $C_{1}$ and $C_{2}$. Let $a, b, c$, and $d$ be the intercepts of the line $x_{0}$ with the circles $C_{1}^{\prime}$ and $C_{2}^{\prime}$ in the inverted plane, $d<c<a<b$. By direct calculation, similar to the proof of Theorem 3 in [3], we obtain

$$
\begin{aligned}
& f_{1}\left(s_{1}, s_{2}\right)=\frac{-a b+(a+b) s_{1}-2 s_{2}^{2}-c d+(c+d) s_{2}}{s_{1}-s_{2}} \\
& f_{2}\left(s_{1}, s_{2}\right)=\frac{2 s_{1}^{2}+a b-(a+b) s_{1}+c d-(c+d) s_{2}}{s_{1}-s_{2}}
\end{aligned}
$$

1) Upper Limit

$\frac{\partial f_{1}\left(s_{1}, s_{2}\right)}{\partial s_{1}}=0$ if $s_{2}=\frac{1}{4}\left((a+b+c+d) \pm\left[(a+b+c+d)^{2}-8 a b-8 c d\right]^{1 / 2}\right) ;$

we have $\frac{1}{4}\left[(a+b+c+d)-[(a+b+c+d)-8 a b-8 c d]^{1 / 2}\right]>c$, and $K$ is a monotonically increasing function of $s_{1}$, for the allowed position $d<s_{2}<c$. Therefore we choose $s_{1}=b$.

$$
\frac{\partial f_{1}\left(s_{1}, s_{2}\right)}{\partial s_{2}}=0 \text { for } s_{2}=b-\left[\frac{(b-c)(b-d)}{2}\right]^{1 / 2} ;
$$

we have

$$
s_{2}\left(K_{\max }\right)<c, \quad \text { if } \quad b-\frac{(b-c)(b-d)}{2}<c,
$$

i.e. $(b-d) \geqq 2(b-c)$ and the bounds $(4 a),(4 b)$ are obtained by substitution of $s_{1}=b$ and $s_{2}=c$ or $s_{2}=b-(b-c)(b-d) / 2$ in (11). The proof for the lower bound is similar and the bounds (4c) and (4d) are obtained by substituting $s_{2}=d$ and either $s_{1}=a$ or

$$
s_{1}=d+\left[\frac{(a-d)(b-d)}{2}\right]^{1 / 2} \text { in (12). }
$$

By inspection of formula (4) it can be seen that the maximum is increased and the minimum decreased by increasing $b$ and $c$ and decreasing $a$ and $d$. If we compare the bounds obtained by leaving the size of the circles $C_{1}^{\prime}$ and $C_{2}^{\prime}$ invariant and changing the position of the line $x=x_{0}$ intercepting the two circles, it can be seen that the largest variation is obtained if the line $x=x_{0}$ passes through the centers of $C_{1}$ and $C_{2}$, and our theorem remains true if we replace the intercepts $a, b, c$ and $d$ by the intercepts of the same circles moved parallel to the $x$-axis so that the two centers of the circles and the two centers of gravity $G_{1}$ and $G_{2}$ lie on the same parallel to the $y$-axis. By inverting this configuration back to the original plane we obtain the configuration of Theorem 3, and Lemma 1 is proved.

The proof of Theorem 4 follows from Lemma 1 by methods similar to the ones employed in [2]. The rational function (1) with zeros in $D_{1}$ and poles in $D_{2}$ is represented as a finite product of rational functions (5) with one pair of 
zeros and poles and the curvature $K$ of (1) is obtained as the finite sum of terms of the form (6). The extreme values of $K$ will be assumed if all the pairs are coincident and the bounds established in Lemma 1 thus hold for all functions (1).

IV. The level curves of harmonic functions. In [3] it was shown that a close connection exists between the level curves of Green's function and lemniscates; in a similar manner the equipotentials of more general harmonic funetions can be shown to possess properties similar to those of rational functions. In this section the properties of the bisectors, normals, and curvature of these equipotentials are derived. As a basis for the proof of these theorems we will use a representation of a harmonic function in terms of its distance to the boundary of a region.

Starting with Green's third identity we obtain the following representation for $u(\xi, \eta)$, harmonic in a region $R$, exterior to a set of smooth curves, $u=0$ on $C_{1 i}, i=1, \cdots, m$ and $u=1$ on $C_{2 i}, i=1, \cdots, n$ at an arbitrary point $P(\xi, \eta) \epsilon R . u$ is continuous in $\bar{R}[5$, pp. 270-272].

$$
\begin{aligned}
u(\xi, \eta) & =\int_{C_{1}} \log r d \sigma-\int_{C_{2}} \log r d \sigma, \\
C_{1} & =\sum_{i=1}^{m} C_{1 i}, \quad C_{2}=\sum_{i=1}^{n} C_{2 i},
\end{aligned}
$$

$r$ denotes the distance from $P$ to a boundary point,

$$
\begin{aligned}
& \text { on } \quad C_{1}-\frac{1}{2 \pi} \frac{\partial u}{\partial n} d s=d \sigma>0, \quad 0 \leqq \sigma \leqq \tau=\int_{C_{1}} d \sigma=\int_{C_{3}} d \sigma, \\
& \text { on } \quad C_{2}-\frac{1}{2 \pi} \frac{\partial u}{\partial n} d s=d \sigma .
\end{aligned}
$$

It follows from the representation (13) that the level curves of our function $u(x, y)$ can be interpreted as a potential and the orthogonal trajectories as lines of force due to a spread of positive matter $\sigma$ on the $C_{1 i}$ and negative matter $-\sigma$ on the $C_{2 i}$, where the total mass is zero and the inverse distance law holds.

If $R$ is an infinite region, integration over a large circle has to be added in the boundary of a region over which the integration is performed and an additional term $u(\infty)$ is to be added to the right-hand side of (13).

With the aid of this representation we can prove an analogue of Bôcher's theorem for harmonic functions. Similarly we obtain

Theorem $1^{\prime}$. Let $u(x, y)$ be a function harmonic in a region $R$, continuous in $\bar{R} . R$ is bounded by mutually disjoint Jordan curves $C_{1}$ and $C_{2}, u=0$ on the $C_{1 i}, i=1, \cdots, m, u=1$ on the $C_{2 i}, i=1, \cdots, n$. Then the perpendicular bisector of a chord joining two points of a level curve

$$
U_{\mu}: u(x, y)=\mu
$$

cannot separate the $C_{1 i}$ and $C_{2 i}$. 
Proof. Let $A\left(x_{a}, y_{a}\right)$ and $B\left(x_{b}, y_{b}\right)$ be two points on (14). Assume $A$ and all the $C_{1 i}$ to the right of the bisector and all the $C_{2 i}$ to the left;

then $\log r_{A C_{1 i}}<\log r_{B C_{1 i}}, i=1, \cdots, m$, $\log r_{A C_{2 i}}>\log r_{B C_{2} i}, i=1, \cdots, n$,

$r_{A C_{1 i}}\left(r_{A C_{3 i}}\right)$ is the distance from $A$ to any point on $C_{1 i}\left(C_{2 i}\right)$,

$r_{B C_{1 i} i}\left(r_{B C_{2 i} i}\right)$ is the distance from $B$ to any point on $C_{1 i}\left(C_{2 i}\right)$.

Substituting in (13), $u(A)<u(B)$ and we obtain a contradiction.

By letting $A$ and $B$ approach each other we obtain the result equivalent to one due to Walsh [5, Cor. 1, p. 277].

Theorem 2'. The normal to (14) cannot separate the boundaries $\alpha$ and $\beta$.

Theorems 3 and 4 also have their analogues for harmonic functions. The curvature of the level curves of harmonic functions can be calculated by means of representation (13). We will choose the point $P(\xi, \eta)$ to be the origin and let the normal to (14) coincide with the negative $y$-axis, and we obtain:

$$
\begin{gathered}
K=\frac{\left[\int_{C_{1}} \frac{y^{2}-x^{2}}{r^{4}} d \sigma-\int_{C_{2}} \frac{Y^{2}-X^{2}}{R^{4}} d \sigma\right]}{\int_{C_{1}} \frac{y}{r^{2}} d \sigma-\int_{C_{2}} \frac{Y}{R^{2}} d \sigma}, \\
r^{2}=x^{2}+y^{2}, \quad R^{2}=X^{2}+Y^{2} .
\end{gathered}
$$

Formula (15) corresponds to (3) for the curvature of the level curve of a rational function and the derivation of the bounds on the curvature can be carried out with integrals replacing the finite sums. This connection enables us to derive the following result on the curvature of (14).

Theorem $3^{\prime}$. Let $u(x, y)$ be a function harmonic in $R$, bounded by smooth curves $C_{1}$ and $C_{2}$. Let all curves $C_{1 i}$ on which $u(x, y)$ assumes the value zero lie in a half plane $Q_{1}$ bounded by a line $q_{1}$. Let all the $C_{2 i}$ be contained in the half plane $Q_{2}$ bounded by $q_{2}, q_{2}$ parallel to $q_{1}, Q_{1} \cap Q_{2}=\varnothing$. Let $P$ be an arbitrary point in the strip exterior to $Q_{1}$ and $Q_{2}$, denote by $A_{1}$ and $A_{2}$ the intersections of the normal $n$ to the curve (14) at $P$ with $q_{1}$ and $q_{2}$. Then the radius of curvature of the curve (14) is numerically greater than the smaller of the distances $P A_{1}$ or $P A_{2}$.

Theorem 4'. Let $u$ be harmonic in a region and assume the value zero on a set of curves $C_{1 i}$ contained in a disc $D_{1}$ and unity on a set of curves $C_{2 i}$ contained in a disc $D_{2}, D_{1} \cap D_{2}=\varnothing . P$ exterior to $\left(D_{1} \cup D_{2}\right)$. Draw the four circles tangent to $D_{1}$ or $D_{2}$ through $P$ orthogonal to the normal to $u(x, y)$ at $P$. Let $A$ and $B$ denote the intersections of the circles tangent to $D_{1}$ with the normal, and $C$ and $D$ the intersections of the circles tangent to $D_{2}$ with the normal. Let the directed distances $P A$, $P B, P C$, and $P D$ be denoted by $1 / a, 1 / b, 1 / c$, and $1 / d$. Then the curvature of $u(x, y)$ must lie between the limits $(4 \mathrm{a})$ to $(4 \mathrm{~d})$.

The method of proof is similar to that of Theorem 4, with integrals replacing the finite sums. 


\title{
BiBLIOGRAPHY
}

[1] M. Bôcher, A problem in statics and its relation to certain algebraic invariants, Proc. Am. Acad. of Arts and Sciences, 40 (1904) 469-484, as quoted in [5, p. 89].

[2] P. Davis \& H. Pollack, On the zeros of total sets of polynomials, TAMS, 72 (1952) 82.

[3] Dorothy Browne Shaffer, Distortion theorems for lemniscates and level loci of Green's functions, Jour. d'Anal. Math., XVII (1966) 59-70.

[4] J. L. WALSH, Lemniscates and equipotential curves of Green's function, Am. Math. Monthly, 42 (1935) 1-17.

[5] J. L. WALsh, The Location of Critical Points, Am. Math. Soc. Coll. Publication, N. Y., 1950.

[6] J. L. WALSH, The circles of curvature of the curves of steepest descent of Green's function, Am. Math. Monthly, 68 (1961) 323-329.

\author{
Fairfield University \\ Fairfield, Connecticut \\ Date communicated: ОстовеR 31, 1968
}

